\title{
Dynamique des pouvoirs locaux liés à la gestion du Marché Central de Parakou
}

\section{Abou-Bakari Imorou}

\section{(2) OpenEdition}

\section{Journals}

Édition électronique

URL : http://journals.openedition.org/apad/436

DOI : $10.4000 /$ apad. 436

ISSN : 1950-6929

Éditeur

LIT Verlag

Édition imprimée

Date de publication : 1 juin 2000

Référence électronique

Abou-Bakari Imorou, « Dynamique des pouvoirs locaux liés à la gestion du Marché Central de Parakou », Bulletin de l'APAD [En ligne], 19 | 2000, mis en ligne le 24 juillet 2006, consulté le 07 septembre 2020. URL : http://journals.openedition.org/apad/436 ; DOI : https://doi.org/10.4000/apad. 436

Ce document a été généré automatiquement le 7 septembre 2020.

Bulletin de I'APAD 


\title{
Dynamique des pouvoirs locaux liés à la gestion du Marché Central de Parakou
}

\author{
Abou-Bakari Imorou
}

1 L'État béninois au milieu des années 1980, comme nombre d'États africains s'était trouvé dans une situation de crise économique. Les manifestations marquant l'échec de la stratégie de développement adoptée par le régime marxiste s'étaient intensifiées. La reconstitution de la structure politique nationale avait donné naissance à des systèmes locaux devant insuffler une sorte de dynamique d'auto-développement régional/local. C'est dans cette atmosphère d'émergence de légitimités locales agissant pour le développement local, au nom d'acteurs qui pensent que leurs préoccupations ne sont plus pris en compte par l'État, qu'est né et a évolué l'idée de la construction d'un marché de classe internationale à Parakou, principale ville du Nord Bénin.

2 Après différentes tractations, la construction du marché a été financée par l'Agence Française de Développement qui a accordé une subvention de 3,5 milliards de FCFA à l'État béninois à cette fin. Cette subvention a été investie dans la perspective de la mise en valeur de certains équipements marchands pouvant générer des revenus pour soutenir la décentralisation. La logique qui a donc sous-tendu le financement de la construction de cette infrastructure est purement économique.

3 Pour atteindre les objectifs fixés, une société de gestion à économie mixte, la SGMP-SEM, a été mise sur pied pour gérer le marché et verser des redevances à la Circonscription Urbaine de Parakou, future Mairie de Parakou. Mais depuis Juillet 1997 que ce marché a été mis en activité, la SGMP-SEM est confrontée à des difficultés de tous genres pour assurer le recouvrement des loyers, principale source de revenu de ladite société. Pire, les usagers estiment les loyers trop élevés et manifestent régulièrement pour leur rabais. L'Agence Française de Développement a considéré que c'est une véritable violation des clauses de la convention de financement et menace de suspendre les autres financements dont le pays doit bénéficier si la situation d'équilibre n'est pas réalisée à la SGMP. Les injections de l'État n'ont pas pu régulariser la 
situation. Cet article est une étude de cas de l'interaction privé - public à travers un chevauchement de logiques différentielles.

Contexte d'émergence du projet du marché de Parakou

4 Le milieu des années 1980 a été marqué au Bénin par une crise économique aiguë. Arrêt de l'engagement des diplômés dans la fonction publique et augmentation du nombre de déflatés, incapacité de l'État à financer des réalisations socio-communautaires et surtout à payer les salaires de ses employés. Les conséquences de cette crise étaient nombreuses et ce, dans tous les domaines de la vie sociale. Cette situation de tension sociale insoutenable a conduit le régime du parti unique d'alors à convoquer la Conférence Nationale des Forces Vives de la Nation de Février 1990. A l'ordre du jour : faire un diagnostic des maux qui minent le développement de la nation béninoise et identifier les nouvelles pistes de sortie de crise.

5 Le désengagement de l'État et la prise de la relève par la société civile ont été constatés. Le discours devenu populaire, disqualifie de manière remarquable l'État jacobin et l'État providence pour ne laisser de l'espace que pour des termes devenus familiers dans le pays, en moins d'une décennie: liberté d'entreprise, auto-emploi, initiative privée, décentralisation, développement à la base, participation, etc. (Sehoueto, 1997 : 10).

6 La situation n'avait guère été soulagée par les injonctions des institutions telles que la Banque Mondiale et le FMI qui ont conduit le Bénin à s'engager dans un programme d'ajustement structurel. L'État Béninois s'est trouvé dans un élan contraire à celui des années 70 où il fallait tout étatiser, tout centraliser et tout contrôler et où, le discours était celui du "comptons d'abord sur nos propres forces" 1

7 La situation de la lin des années 1980 était celle du désengagement de l'État, de la "décentralisation". Les entreprises autrefois étatiques avaient commencé par être privatisées suivant un programme indiqué par les bailleurs de fonds. La stratégie étatique de redressement de la situation a été bien identifiée par Nassirou Bako Arifari (2000) quand il écrit "... le régime était alors dans une double quête, d'une part, de relais plus dynamiques pour assurer la remobilisation et le réarmement moral des populations à la base pour les engager dans des actions de développement et, d'autre part, de ressources extérieures complémentaires pour sa reproduction interne".

8 Ainsi des actions sont menées un peu partout dans le pays, dans le sens de la prise en charge effective des populations par elles-mêmes. Certaines de ces actions sont menées par des associations ou des Organisations Non Gouvernementales (ONG) sur fond de philanthropie. Dans ce cas l'État n'intervient pas. Un nombre important d'écoles, de centres de santé, de latrines publiques, de fontaines publiques a ainsi vu le jour.

9 D'autres actions par contre sont des initiatives des populations qui sollicitent l'appui de l'État pour la réalisation d'infrastructures communautaires. C'est sur cette dernière logique de développement que des populations se font aider par l'État dans la recherche des fonds pour la réalisation d'un projet de réfection du marché international de Parakou.

10 En effet le marché international de Parakou est le résultat d'un long processus par lequel des idées se sont succédé pour donner naissance à un chef d'œuvre architectural qui contraste véritablement avec son milieu d'implantation.

11 Une présentation sommaire et diachronique du projet nous montre que tout avait commencé en 1985, année durant laquelle il était demandé de reconstruire le marché 
central pour lui permettre d'être conforme à la taille de la ville en plein essor démographique. Cette idée a vu le jour à un moment où l'État avait commencé par ressentir les conséquences de la crise économique. Il lui était donc impossible de financer une telle réalisation. A la suite d'une longue période elle a été mise en veilleuse puis réactivée avec la remise en marche de l'économie nationale à partir de 1990.

12 L'Association de Développement de Parakou dénommée "Timbi" en a fait une préoccupation prioritaire et le gouvernement l'a aidée à trouver un bailleur de fonds : la Caisse Centrale de Coopération Economique (devenue Agence Française de Développement depuis 1998). Le marché a donc été construit sur la base d'une convention de financement signée entre le Bénin et la France. L'une des clauses de la convention est la création d'une société anonyme pour gérer les marchés. Les règles et procédures de fonctionnement ont été dictées à la structure de gestion sur la base de critères de performance, car, a-t-on souligné, le marché doit être rentable à la collectivité, il doit entre autres constituer la principale source de revenus de la future municipalité de Parakou. Le marché de Parakou, rendu opérationnelle mercredi 18 juin 1997, est devenu depuis sa restructuration et son extension, le théâtre d'affrontement et de confrontation. Nous pouvons en effet observer deux facteurs essentiels. D'une part, le problème du marché international de Parakou résulte du décalage manifeste entre les visions logiques et les prévisions qui ont prévalu à l'élaboration du projet de réfection et aussi les logiques et pratiques d'acteurs qui agissent in situ. D'autre part, le problème du marché est moins lié à la gestion des ressources matérielles et financières qu'à celui de la gestion d'acteurs socio-économiques aux intérêts le plus souvent divergents. On peut dès lors se demander comment se sont constitués les réseaux locaux, et quelles stratégies ils utilisent pour court-circuiter l'action publique administrative?

13 Il s'agira d'analyser à partir de ce cas les formes de gestion des infrastructures socio-économiques locales en prenant en compte les articulations entre le privé et le public et surtout à la lutte pour la privatisation du public par des acteurs locaux. Il faut pour ce faire présenter la structure qui a été l'objet de notre analyse ainsi que les interactions observées autour d'elle.

Le fonctionnement de l'ancien marché

14 Le site actuel du marché international de Parakou était une vaste cour d'escale des caravanes venant des régions septentrionales. Cette cour faisait face à la concession du Baparapé de Parakou qui était le représentant des communautés étrangères auprès des autochtones en général et auprès du chef supérieur de Parakou en particulier. C'était donc un lieu de troc entre les étrangers et les autochtones. Le marché a un chef traditionnel qui s'occupe des cérémonies en vue de la paix des usagers. Ce marché a évolué suivant la croissance démographique de la ville. Les agglomérations à l'est du site ont pris le nom de Yebu Beri qui est le nom local du marché et signifie grand marché en Dendi. Ce nom est donné par opposition à Yara-kinnin qui veut dire "Petite cours" et qui est le nom des agglomérations situées à l'ouest du marché et qui avaient de petites cours d'échange qui ont par la suite été supprimées. L'évolution du grand marché ne s'était pas faite suivant une réglementation stricte avant 1958, année au cours de laquelle il y a eu la construction d'une quinzaine de hangars sur le site. Cet acte en même temps qu'il a accéléré la croissance du marché, a constitué le point de départ du paiement des taxes par les usagers du dit marché. 

cause de l'influence de la proximité d'un assez grand nombre de boutiques modernes et de commerces importants. Son intérieur était un lieu d'exposition des étagères en bois ou en métal, des étagères en palmes tressées, des paniers et bassines. On y voyait aussi des expositions par accrochage ou suspension sur des traverses en bois ou des expositions au ras du sol avec ou sans support. C'est ce spectacle qui ne serait pas de nature à offrir une bonne image de la ville aux étrangers qui, ajouté à la faiblesse de recettes mensuelles due au recouvrement des taxes sur le marché, ont justifié la pertinence de l'idée de sa réfection pour lui donner un visage plus moderne et pour accroître les recettes qu'il génère.

Le projet de réfection du marché responsables de la province d'alors, qui aujourd'hui correspond au département du Borgou (un des six départements que compte le pays; la décentralisation portera ce nombre à 12). L'initiative a été soutenue par les responsables de l'Association de Développement qui ont sollicité le soutien de l'État pour le financement. En 1987 déjà l'avant-projet détaillé qui faisait état de la nature des matériaux il utiliser et des bâtiments à réaliser était achevé. Mais le projet n'a pas démarré à temps il cause d'un certain nombre de problèmes : le site devant abriter l'infrastructure et le bouclage du financement. Le bouclage du financement a en effet, été un grand problème que devaient résoudre les promoteurs du projet. Après plusieurs tentatives auprès de la Banque Ouest Africaine de Développement (BOAD) et au Fonds Africains de Solidarité, c'est finalement la Caisse Centrale de Coopération Economique qui a offert les meilleures conditions d'octroi du crédit nécessaire à la réalisation du chef-d'œuvre. L'accord de principe de la Caisse avait été concrétisé par deux missions sur les lieux courant 1989.

17 A la suite de ces missions et sur la base des rapports produits, la Caisse a été autorisée par son comité d'États étrangers à consentir au Bénin un crédit de $35.000 .000 \mathrm{FF}$ soit 1.750.000.000 FCFA à cette époque. Ce montant représentait la totalité des fonds nécessaires à la réalisation du projet. En 1990 lors du sommet France - Afrique de la Baule, le Président Mitterrand avait exigé qu'il n'y ait plus de crédit français il l'endroit des pays africains dont les économies étaient en difficulté, mais plutôt des subventions. Ainsi le crédit accordé au Bénin était devenu une subvention. La dévaluation du francs CFA a fait passer l'enveloppe de 1,750 milliards à 3,5 milliards. Une convention de financement W 5823600514 OM en 17 articles a été signée entre le Bénin et la France. Cette convention définit les conditions, les objectifs et les clauses du financement et a été enregistrée à Cotonou le 8 juillet 1992. L'une des clauses de la convention précise que l'État s'engage à prendre des mesures nécessaires pour que le bénéficiaire mène une politique tarifaire sur le marché de Parakou.

18 La Caisse Centrale s'est enfin réservée le droit d'ajourner et même de rejeter définitivement toute demande de versement si la République du Bénin ne respectait pas l'un quelconque des engagements contractés envers la Caisse Centrale, soit au titre de la convention, soit au titre de tout autre acte. Après la signature de la convention, il était question de démarrer les travaux quand le problème du site devant abriter l'infrastructure s'était posé. La question était de savoir s'il fallait déplacer le marché et le construire sur un autre site ou non. Elle a nourri une vive polémique tant du côté des bailleurs que celui des bénéficiaires. Les uns présentaient des arguments 
environnementaux pour soutenir la nécessité de construire le marché ailleurs tandis que les autres présentaient des arguments culturels pour montrer qu'on ne déplace pas un marché comme on peut déplacer les autres infrastructures.

Mais il cause des expériences que l'Agence Française de Développement (AFD) a sur les échecs des marchés déplacés, l'unanimité a finalement été faite sur la construction du nouveau marché sur le site de l'ancien. C'est dans ces conditions qu'a été réalisé le projet qui a finalement pris le nom de "projet de restructuration et d'extension du marché de Parakou". Pour gérer l'infrastructure conformément aux clauses de la convention, une Société dénommée "Société de gestion des marchés de Parakou (SGMP)" a été mise sur pied. Cette Société à économie mixte a été l'objet d'un montage institutionnel définissant comment elle doit être gérée. C'est avec la SGMP que les usagers doivent signer le contrat de bail. Et c'est à la SGMP qu'il revient de payer les redevances mensuelles à la Circonscription Urbaine de Parakou (CUP), propriétaire de l'ouvrage. L'Administration locale n'a pas à gérer l'ouvrage, mais elle a le devoir d'assister la SGMP à faire face aux difficultés auxquelles elle se trouverait confrontée dans la gestion du marché.

Attribution des places et régimes de privilèges

Le tout premier problème auquel la SGMP a été confrontée est celui de l'attribution des places du marché (756 étals, 275 boutiques et 3 maquis). C'est ensuite que c'est posé le problème des prix des places. La question de l'attribution des places du marché remonte au 10 juillet 1997. Les consultants du cabinet français C2G-Conseil avaient été chargés par l'AFD d'étudier les modalités d'attribution des places dans le marché. A partir des analyses faites des différentes propositions, les consultants avaient indiqué le critère du "plus offrant" comme le principal critère d'attribution. Selon ce critère une boutique mise en location doit être attribuée au plus offrant.

21 Mais au moment où il fallait procéder à l'installation des usagers, ceux-ci par l'intermédiaire de leur syndicat qui venait d'être créé, avait refusé le critère du plus offrant qu'ils avaient estimé exclusifs de certaines catégories d'usagers. Ils avaient menacé de ne pas intégrer le marché et contraint la SGMP il la négociation. Lesdites négociations avaient conduit à l'implication des syndicalistes dans l'attribution des places. Ceux-ci avaient bénéficié du privilège de procéder à l'attribution des boutiques intérieures et des étals. Les boutiques extérieures devaient être attribuées par les membres du Conseil d'administration (CA) de la SGMP.

22 Les membres du CA avaient opté pour la récompense de ceux qui ont œuvré d'une manière ou d'une autre à la réalisation du projet. Ainsi les autorités locales (même celles qui sont venues après la construction) les personnalités de la place et les membres du CA eux-mêmes, avaient tous bénéficié d'au moins une boutique chacun. Donc des 41 boutiques extérieures, aucune n'a été officiellement attribuée à un commerçant.

$\mathrm{Au}$ niveau des syndicalistes l'attribution a été faite sur la base de critères non élucidés. Le constat qui s'impose est que dans ce marché, après les attributions, certaines couches socio-ethniques n'ont pas officiellement trouvé de place. Les vendeurs de friperie par exemple, bien qu'ils soient les plus actifs usagers du marché, n'ont pas été retenus officiellement; et ils sont tous Nigériens. Il en est même pour les Yoruba et les ressortissants des régions méridionales du Bénin. L'une des conséquences de cette situation est la sous-location des places dans le marché.

La Sous-location des places 
La sous-location est une situation vécue en violation du règlement intérieur du marché. En effet, en son article 2, aliéna 3, le règlement intérieur souligne que les emplacements "... ne peuvent être occupés que par les bénéficiaires et leurs employés. Ils sont strictement personnels et ne peuvent, en aucun cas être vendus, sous-loués, amodiés sous quelle que forme que ce soit ou présentés en garantie".

Face à l'exclusion dont ils ont été l'objet au moment de l'attribution des places, les Nigériens en particulier, ont adopté une stratégie qui leur permet de trouver des places dans le marché : la sous-location. Ce fait a été favorisé par l'attribution des places à des individus qui ne mènent aucune activité commerciale. La sous-location est aussi le fait de l'attribution de plusieurs boutiques à une même personne, surtout les responsables syndicaux. Ces derniers en effet, puisque c'est eux qui ont procédé à l'attribution de certaines boutiques, se sont attribué chacun deux à trois boutiques. Pour se rendre compte de l'ampleur du phénomène de sous-location, il faut partir du nombre réel de Nigériens qui occupe des boutiques. Leur nombre avoisine la centaine.

A l'issue des différentes revendications de la communauté nigérienne de Parakou, onze boutiques ont été cédées à cette dernière. Ainsi. il ne devrait y avoir dans le marché que onze nigériens attributaires de boutiques. A la question de savoir pourquoi la SGMP ne peut pas appliquer les textes contre le phénomène, son Directeur Général estime qu'il n'y a pas d'argument juridique car il y a une complicité de fait entre les acteurs de la sous-location. Les Nigériens se présentent toujours comme étant des employés des propriétaires des boutiques qu'ils occupent. L'impuissance de l'administration à faire respecter les textes a fait que les acteurs locaux sont devenus si forts que leur désir est respecté en violation de toutes les prévisions faites dans le sens de la rentabilisation de cet équipement marchand qu'est le Marché International.

Privatisation du bien public et fixation des prix des boutiques du marché

Dans les études qui ont conduit à la fixation des prix, les consultants sont partis de la surface commerciale qui sera mise en valeur. Les surfaces ont donc été décomposées en étals et en boutiques auxquels ont été affectés des montants de loyer, par type de surface unitaire de vente. Dans les montants estimatifs, la surface utile de vente envisagée étant d'une superficie de $10530 \mathrm{~m} 2$, les loyers ont été fixés à 28 FCFA/m2/ jour pour les étals et à $32 \mathrm{FCFA} / \mathrm{m} 2 /$ jour pour les boutiques.

Ces tarifs avaient été estimés par surface en fonction des charges prévisionnelles de fonctionnement de la Société de gestion. Ces charges se résument suivant le contrat d'affermage, aux obligations de la SGMP vis-à-vis de la collectivité, à la constitution de fonds de réserve pour le renouvellement des installations en cas d'amortissement, aux différentes taxes, au dividende à verser aux actionnaires et aux dépenses relatives à l'eau, l'électricité, à la masse salariale des travailleurs de la société. Ainsi les prix étaient fixés à $12000 \mathrm{~F} /$ mois pour la boutique la moins chère à $90000 \mathrm{~F} / \mathrm{mois}$ pour la plus chère, et à $200 \mathrm{~F} /$ jour pour les étals. C'est sur ces prix de base que devrait s'appliquer la loi du "plus offrant".

Les syndicalistes ont combattu ces prix qu'ils ont trouvés trop élevés et les ont réduits à la moitié de leurs valeurs prévues. Les grèves de protestations des prix ont entraîné la mise en scène d'un nombre important de médiateurs pour dénouer la crise. La situation était telle que l'Administration avait renoncé à ses intérêts économiques pour sauvegarder la paix sociale et par-delà les intérêts politiques. 
30 Il y avait là une double privatisation du bien public. D'une part, les responsables de l'administration locale ont offert le marché aux forces locales pour éviter d'être impopulaires. D'autre part, les syndicalistes et tous ceux qui ont sous-loué bénéficient de cet avantage évident : les diminutions des prix officiels n'ont pas d'incidence réelle sur les prix de sous-location. De sorte que ces derniers réalisent un bénéfice sur les revenus de la société de gestion et par-delà elle, sur la collectivité. La situation a fait l'objet de dénonciation mais toutes les tentatives de récupération de ce manque à travers la légère élévation des prix notés ces derniers mois, ne sont pas à la hauteur du phénomène. Il y a une forte politisation des problèmes du marché et un chevauchement complexe de plusieurs logiques et stratégies. La puissance publique s'est affaiblie et on a une dynamique locale qui fonctionne sur le registre de menace de défection politique. Le projet du marché comme champ de confrontation des logiques différentielles Les projets de développement se présentent souvent sous forme de schémas classiques d'intervention de l'extérieur. Parfois c'est un financement qui est accordé à une localité par un "partenaire au développement". Le "partenaire" oblige les bénéficiaires à acheter le matériel, et la compétence nécessaire chez lui, pour la réalisation du projet. Cette pratique correspond à celle de l'aide liée. On note aussi suivant une autre logique, celle du respect de la souveraineté des deux partenaires, la mise en relation du fonctionnement à partir d'une aide que l'un des partenaires accorde à l'autre. Le bénéficiaire est autonome dans la gestion du projet au niveau local. Tout ce qui vient de l'extérieur se limite aux visites d'évaluation. Dans ce cas qui est rare, les bailleurs n'interviennent pas dans la gestion.

Dans l'un ou l'autre cas et même dans d'autres schémas non énumérés dans le présent travail, le projet de développement est un espace-temps, un dispositif qu'on greffe à un environnement donné et dont la disparition crée un équilibre préalablement rompu. Qu'est-ce qui en fait rompt l'équilibre du milieu où se greffe le projet? Pour reprendre les termes de J.P. Olivier de Sardan (1995) : "Autour d'un dispositif de développement quelconque se confrontent de multiples logiques et stratégies, du côté des agents du dispositif comme du côté des populations dites 'cibles"'. Suivant la nature du projet et les différentes étapes qui ont caractérisé sa conception, et le lieu de son implantation, les populations cibles, qui en fait sont elles aussi des actrices, ont des comportements variés. Parfois, leurs comportements contrastent avec l'idéal qui sous-tend le projet aux yeux des bailleurs. Cette situation peut s'analyser à partir d'un point de vue qui renvoie à des logiques d'intérêt différentes et donc à des positions différentes par rapport au projet.

33 Le projet de réfection et d'extension du marché central de Parakou intervient dans un milieu qui n'en est pas à sa première intervention. Les populations de Parakou ont été témoins d'un certain nombre d'actions sous forme de projet. Une fois à terme le bailleur de fonds disparait et le résultat de l'intervention demeure et est géré par les locaux. Il en a été ainsi pour la construction de nombreuses écoles, des centres de santé, des puits à grands diamètres, etc. Toutes ces réalisations faites sous forme de don ont construit dans la logique des populations locales, une façon de comprendre les projets de développement. Même si le plus souvent on demande une participation des populations avant de subventionner la réalisation d'un ouvrage, une constante notable est qu'une fois l'intervention terminée, les populations ne voient plus le bailleur s'ingérer dans la gestion ou faire un quelconque appel à l'ordre. En terme d'ingérence réelle et manifeste, de changement par rapport à la façon classique des projets de 
développement, celui de la réfection du marché de Parakou représente un cas assez pertinent.

34 C'est dès la base, à la signature de la convention de financement que les bailleurs ont manifesté leur désir d'avoir toujours un regard dans la façon dont le projet et son résultat seront gérés. Nous référant à la logique des bailleurs, on doit souligner que le projet de réfection de ce marché rentre dans la mise en valeur des équipements marchands pouvant octroyer à la CUP, future Mairie, une source de revenus. Cette logique est partagée par le gouvernement par l'intermédiaire du MISAT qui a inscrit la CUP comme une circonscription à statut particulier c'est-à-dire à cheval entre le système déconcentré et le système décentralisé.

Il s'agit, selon celle logique, de faire en sorte que le marché central soit géré de façon à générer des revenus pouvant servir à financer d'autres réalisations socio-communautaires. Pour les bailleurs c'est là une aide qui, si elle est bien gérée, peut permettre de se passer de l'aide. Mais il faut retenir que cette logique n'est partagée qu'entre les bailleurs, les autorités politiques et administratives nationales et locales, et les promoteurs. Celle question n'a pas été discutée avec les populations bénéficiaires. Bien au contraire, les bénéficiaires avaient toujours été représentés par l'administration locale et les membres de l'association de développement qui sont supposés représenter les communautés locales et les usagers du marché, sans avoir un contact réel d'explication avec eux. Mieux, le discours tenu devant les bénéficiaires est celui du don, du soutien des bailleurs qui ont le souci de soulager les peines des usagers tout en donnant à la ville un visage digne de son rang sur le plan national et régional. Le couronnement dans le sens de ce discours est l'appel lancé par les promoteurs pour amener les locaux à acheter des actions dans le capital social de la société à économie mixte qui devait assurer la gestion du marché. "C'est notre chose, c'est à nous qu'il revient de le gérer. Ni l'État, ni aucune autre institution n'aura rien à nous dicter. Par conséquent, on aura assez de bénéfices si on est actionnaire car le marché ne souffrira d'aucune insuffisance de gestion". Ce sont là les termes qui pourraient résumer le marketing fait pendant la constitution de la SGMP; marketing au cours duquel on fait remarquer aussi aux usagers que c'est à eux de désigner les gestionnaires du joyau.

C'est ce qui pourrait certainement expliquer la dynamique qui a été observée à la veille de l'installation des usagers dans le nouveau marché et qui a conduit à une stratification de l'ensemble des usagers en groupes particuliers dont le plus notable est celui du Syndicat des Revendeurs et Revendeuses des marchés de Parakou. Ce groupe est entré en scène avec les grèves de revendications et soupçonne les autres de mal gérer le marché et de vivre aux dépens d'eux (les usagers). La fonction première qui a sous-tendu la construction du marché est entrain de disparaître au profit d'une autre logique, celle du social. Les usagers forts de l'expérience qu'ils ont des réalisations des projets, estiment que ce qu'on leur demande est trop, les bailleurs à leurs yeux tiennent un double langage.

"On ne peut pas dire qu'on nous a donné le marché et nous exiger tant d'argent à donner par mois. Le loyer est trop élevé si c'était un crédit à rembourser, la cherté du loyer s'expliquerait. Mais maintenant on ne comprend pas ce qui se passe, tout est flou", s'est exprimé un responsable syndical (SGMP) qui est perçu comme proche du pouvoir. La SGMP est perçue par les autres groupes organisés autour des syndicats comme un groupe voulant bénéficier tout seul des avantages du projet. Il est le seul à 
être officiellement en possession des textes et à donc veiller sur leur respect par tout le monde.

Mais il faut rappeler que tout se passe dans une atmosphère où tous les acteurs ne sont pas au même niveau d'information. Dans un premier temps, le soulèvement des usagers avait donc été perçu par eux comme une action contre la SGMP qui tenait au système tarifaire et qui avait fait des frustrés pendant l'attribution des places dans le marché. La SGMP, dans son souci de faire respecter les textes, a donc été soumise à des épreuves. Les revendications sont allées jusqu'à exiger et obtenir la démission du directeur de la SGMP jugé par les usagers comme la source de leurs difficultés. Cependant la crise semble avoir donc sa source ailleurs.

L'administration locale entre deux logiques économique et politique

Le contrat d'affermage qui a permis à la CUP de confier la gestion du marché à la SGMP, indique que l'une des obligations de la CUP est d'assister la SGMP en lui facilitant l'exercice de sa responsabilité. Mais pendant les crises qui ont secoué et qui continuent de secouer la SGMP pas intermittence, la CUP s'est inscrite dans une double logique en cherchant à opérer une juxtaposition du politique et de l'économie. Tous les usagers ont eu des contrats individuels avec la SGM ? Et quand une partie contractante n'est plus d'accord avec les clauses contractuelles, elle est libre de le résilier. Il est important de souligner qu'il n'y a jamais un contrat collectif signé entre les usagers et la SGMP. En outre, les textes qui régissent le fonctionnement du marché n'ont pas prévu l'existence des syndicats des usagers et par conséquent n'ont pas défini la nature des relations qui pouvaient exister entre syndicats et SGMP.

La CUP, principale bénéficiaire de la bonne gestion du marché suivant le système tarifaire retenu, avait la capacité d'intervenir pour rétablir l'ordre. Le moment s'y prêtait car pendant que les usagers protestaient contre les prix, il y avait d'autres commerçants et commerçantes qui se plaignaient de ce qu'ils n'avaient pas eu de place dans le marché et étaient prêts à la prendre à n'importe quel prix. Reprenant les termes de Max Weber, Hyden (1993) définit la puissance comme étant la "probabilité que, au sein d'une relation sociale, un auteur se trouvera en position favorable pour accomplir sa volonté en dépit de toute résistance, quelle que soit la base sur laquelle repose cette possibilité".

41 Nous situant dans ce sens donné au mot "puissance", il est évident que le comportement de la CUP avait constitué une perte de sa puissance en faveur des groupes qui se sont organisés pour changer les règles estimées contraignantes. Dans ces conditions la question des prix des loyers au marché est devenue l'objet d'une mobilisation politique. La logique économique qui a été à l'origine de tout ce qui a été fait, s'est trouvée reléguée au second rang. Comment la crise a-t-elle été gérée pour qu'on en arrive à une telle situation pourrait-on se demander?

Face au soulèvement des usagers, la CUP conformément aux textes a été appelée à la rescousse pour aider la SGMP dans la résolution du problème. Mais il y a eu absence de décision politique rigoureuse de la part de la CUP pour faire respecter les textes qui régissent le fonctionnement du marché. Par rapport à l'évidence du manque de négociation sociale pour obtenir l'adhésion des usagers dans le principe des prix qui a caractérisé les travaux sur le marché, la CUP a voulu impliquer d'autres acteurs locaux pour jouer aux médiateurs. C'est ainsi que le cercle des acteurs et des logiques a augmenté pour occulter totalement la logique économique de départ. Cet état de chose 
a complexifié la crise du marché au point où il est devenu presque impossible à la CUP de prendre une décision non négociée au préalable. Ce dont elle était capable au départ.

La situation est telle qu'on est tenté sans trop grand risque de se tromper de dire que la représentation locale de l'administration étatique a balancé du côté des usagers. On veut la résolution du problème mais on craint la réaction des usagers. Cela explique l'affirmation du chef de la Circonscription Urbaine de Parakou quand il dit: "Je suis d'accord pour augmenter les tarifs mais si cela remet en cause la paix sociale, je vais baisser". On est dans une logique de conservation du capital et politique affectif dont on dispose dans le milieu.

Médiation et expression populaire

Dans l'incapacité de régler les problèmes du marché de la CUP a demandé la médiation d'un certain nombre de personnes dont les membres de l'association des sages et notables de Parakou. De façon traditionnelle, le rôle qu'on confie aux médiateurs c'est de s'entremettre pour faciliter un accord entre deux parties. Il s'agit de concilier des parties qui s'opposent sur un certain nombre de questions. Dans le cas du marché. les médiateurs utilisés pour concilier les points de vue sont estimés plus aptes à faire face à la furie des syndicats, à mieux jouer sur les sensibilités pour amener les uns et les autres à comprendre la nécessité de dénouer la crise pour permettre aux activités commerciales de la ville de se dérouler. Il faut rappeler que pendant les grèves les syndicats des revendeurs et revendeuses adoptent un comportement de blocage total des activités commerciales dans toute la ville. Ils vont jusqu'à perturber la venue des produits vivriers des villages environnants. Ils ferment systématiquement les marchés de quartier et tout vendeur qui viole la règle s'expose à des représailles et même parfois à des sévices corporels.

La mission confiée aux médiateurs dans ces conditions était de tout mettre en œuvre pour en finir avec le blocage du tissu commercial. Ils devaient eux-mêmes faire face à la colère des revendeurs qui avaient estimé que c'est aux yeux et au su des sages et notables de Parakou, des membres de Timbi, qu'on leur applique, à eux, le plus haut prix de tous les marchés de la sous-région. "Si vous vous taisez, nous on va céder, on ne pourra plus continuer par vendre dans le marché et ceux-là qui viendront vendre, vous appliqueront des tarifs pouvant leur permettre de payer les loyers de la SGMP". Les responsables syndicaux avaient réussi à faire comprendre aux médiateurs que la lutte qu'ils menaient était une lutte pour la survie collective et non simplement pour leurs intérêts.

46 L'argument opposé, celui des représentants de la SGMP, était relatif à la sauvegarde de l'honneur en respectant la parole tenue au bailleur, à la nécessité de réaliser l'équilibre financier pour permettre à la ville de bénéficier d'autres subventions de la part des bailleurs. Il fallait tout mettre en œuvre pour ne pas décevoir les bailleurs. Mieux, il fallait selon eux tout faire pour prévoir des ressources pouvant permettre de faire face à l'amortissement du chef-d'œuvre pour que celui-ci ne tombe pas en ruine. Les éléments avancés par la SGMP pour justifier la nécessité de maintenir tout au moins l'équilibre, même s'il n'y a pas de bénéfice à dégager, renvoient à une préoccupation lointaine. Les bailleurs de fonds ne sont pas établis à Parakou, les financements à sauvegarder sont futurs. La préoccupation brûlante est la normalisation des activités commerciales.

47 Cette conclusion des médiateurs avait une conséquence, celle de convaincre la SGMP pour qu'elle accepte les doléances des revendeurs afin de dénouer ne serait-ce que 
momentanément la crise. La prise de position des médiateurs en faveur des usagers ne s'explique pas seulement par les arguments avancés par les responsables syndicaux. Cette prise de position semble à notre avis s'expliquer aussi et surtout par l'implication dont certains médiateurs avaient été objets pendant l'attribution des places. En effet souvenons-nous de ce que pendant l'attribution des places du marché, les boutiques externes avaient été attribuées aux membres du conseil d'Administration de la SGMP et à certaines personnalités de la ville. Les conseillers et hautes personnalités locales à qui des places ont été attribuées ne sont pas pour la plupart des commerçants. Ils ont donc cédé ces places à des proches parents ou amis, au nombre desquels il y a des gens qui ne mènent pas des activités commerciales à la hauteur des boutiques qu'ils occupent. La conséquence évidente, c'est qu'ils les trouvent trop chères. Il y a donc eu à ce niveau des débats sur le marché, l'émergence d'une instance acquise à la cause des revendeurs et qui négocie pour la diminution des prix plutôt que toute autre solution. Cette instance locale avait occupé pour la suite des négociations une position de "substitut à la confrontation" (Lavigne Delville, 1994). Ce sont les membres de cette instance qui ont compris les usagers, qui connaissent mieux leur problème et qui peuvent les défendre au besoin devant les autres instances. Ce qui n'est pas conforme au rôle qui leur était dévolu.

Avec cette tournure, les donnés changent. Ce n'est plus seulement les syndicats qui réclament la diminution, mais au nom du rétablissement de l'ordre socio-économique les associations des sages et notables et Timbi aussi la réclament de façon à peine voilée. Ni les CUP, ni la SGMP ne pouvaient s'y s'opposer.

Toute analyse faite, on remarque que la CUP n'a pas voulu prendre la responsabilité d'encourager la violation d'une convention signée par le Gouvernement. La logique qui consiste à impliquer d'autres instances pour trouver une solution, nous semble une "théâtralisation" du jeu politique. La mise en scène consiste à ne pas permettre aux "supérieurs" de trouver des arguments pour accuser l'exécutant d'avoir fait le jeu des locaux.

Le Président du CNSGMP après avoir apprécié le comportement des usagers face au problème des prix, n'a pas voulu continuer à se coller aux statuts légaux au point de voir son autorité compromise. C'est tout naturellement qu'il a conduit la SGMP à se plier à la volonté "populaire" pour qu'en homme politique, il ne fasse pas les frais d'une quelconque rigueur par rapport au respect des textes et des données économiques. Il a aussi glissé sur le terrain politique. Cela explique sa déclaration quand il dit : "je ne sais pas comment l'Agence Française de Développement peut nous fixer de tels prix à Parakou" 2 .

51 Le "nous" employé par le président en ce moment de la crise, rentre dans une stratégie de recherche de filons identitaires pour montrer qu'il n'est pas différent des usagers qui avaient commencé par le contester. Une fois encore il a cherché à faire bloc avec les siens contre les "autres".

La corruption dans le système

52 Quand nous concevons la corruption comme moyen que l'on emploie pour faire agir quelqu'un contre son devoir. sa conscience, force nous revient d'affirmer que dans les problèmes du marché, la corruption a une part non négligeable. Ce n'est en effet, que par le fait de la corruption qu'on peut expliquer les problèmes de la sous-location et de la diversité des prix pour les boutiques de même nature. 
53 La Direction Générale a conscience de l'existence de la sous-location. Elle sait également que c'est une pratique qui est faite en violation du règlement intérieur du marché. Mais elle n'a pu rien faire de façon manifeste et décisive pour en finir. S'agissant de la responsabilité des directions générales et leur conseil d'administration, deux hypothèses s'imposent à notre analyse : soit ils sont impliqués dans la pratique et donc ils y tirent leurs intérêts, soit ils sont des observateurs impuissants d'un phénomène dont les responsables se trouvent à un niveau de positionnement qui les protège de toute bousculade ou usent de subterfuge qui les protège. Concernant les usagers, il y a aussi deux hypothèses. Soit dans les relations entre propriétaires de boutiques et utilisateurs, on note un trafic d'influence et de menace des premiers sur les seconds, ce qui oblige à se garder de reconnaître qu'ils sont sous-locataires, soit c'est une relation basée sur l'intéressement des uns par les autres. Dans tous les cas, quelqu'un agit contre son devoir vis-à-vis de la société de gestion. Il en résulte un manque à gagner pour la société. Etant donné que c'est évident que ceux qui ont plusieurs boutiques au point de sous louer certaines ou ceux qui ont bénéficié de boutiques alors qu'ils ne sont pas des usagers sont socialement bien placés, on pourrait conclure qu'ils jouent un rôle d'activiste pendant les crises car la diminution des prix les arrange. Plus bas sont les prix officiels, mieux ça vaut pour eux. Mais les hypothèses sont d'autant plus vraisemblables que certains propriétaires sont dans un jeu de médiation.

54 Le mécanisme de ce phénomène est d'autant plus complexe que les utilisateurs des boutiques ont choisi de se présenter comme des employés pour garder leur boutique plutôt que d'accepter qu'ils sont sous-locataires pour que la boutique leur soit officiellement attribuée. Les quelques uns que nous avons pu mettre confiance et qui nous ont affirmé qu'ils sont en sous-location nous ont confié que ce serait une grosse erreur pour eux de dénoncer ceux qui les ont aidés à avoir une boutique quand les autorités officielles les en avaient privés. Le phénomène semble être aussi sous-tendu par un contrat de confiance.

Mais il est si enraciné et complexifié qu'il est difficile d'apprécier sa véritable envergure.

Autochtonie comme stratégie d'appropriation de privilèges et d'exclusion

56 Les lieux communs sur le caractère référentiel du mot étranger pour trancher une dispute ou pour amener les acteurs à se positionner en faveur de telle ou telle partie sont réguliers à Parakou. Mais un fait est de constater que cette régularité ne cache pas du tout la complexité, ni parfois à la limite, l'ambiguïté liée à la notion. Il n'est pas toujours facile de dire qui est autochtone à Parakou. Néanmoins, il serait bon de se pencher sur les situations auxquelles renvoient l'utilisation de cette notion et les raisons de son usage.

57 Le montage financier du marché a prévu, pour des raisons de rentabilité économique, que les places soient attribuées aux instances acquises à la cause des revendeurs et qui négocient pour la diminution des prix plutôt qu'à toute autre solution. Cette instance locale avait occupé pour la suite des négociations une position de "substitut à la confrontation". Ce sont les membres de cette instance qui ont compris les usagers, qui connaissent mieux leur problème et qui peuvent les défendre au besoin devant les autres instances. Ce qui n'est pas conforme au rôle qui leur était dévolu. Avec cette tournure, les donnés changent. Ce n'est pas de l'attribution des places qu'il s'agit. Mais une fois le marché achevé, la première proposition a été contestée par la quasi-totalité 
des usagers de l'ancien site qui ont estimé qu'ils sont prioritaires et que l'application du principe du plus offrant les exclurait car ils ne pensent pas pouvoir supporter la concurrence rude des "envahisseurs" .

58 Le second schéma a été contesté par les nouveaux venus qui ont estimé que cela pourrait avantager les Nigériens qui étaient les plus nombreux parmi les usagers de l'ancien marché. Le discours avait donc été celui de ne pas opter pour un schéma qui va avantager les étrangers. Ce discours, semble-t-il, a influencé le conseil d'administration de la SGMP ou l'a alors renforcée dans son option. D'autres critères officiels étaient venus compléter le critère qui privilégie les anciens usagers. En dehors de l'ancienneté, le second critère qui était important est celui de l'origine. Et il était exigé de la préciser dans la demande adressée à la SGMP.

Aucune précision officielle n'est donnée sur la façon dont l'articulation entre les deux critères a été faite. Toujours est-il que les étrangers n'ont pas pu avoir officiellement de place. Les Nigériens sont restés sur le site provisoire et les ressortissants du sud au marché Dépôt.

60 Une analyse approfondie de ces données n'épargne pas les autorités locales dans l'organisation de cette exclusion. Même si elle n'est pas officiellement systématisée, elle est vécue et constatée. Il suffit de faire le rapport entre le nombre des étrangers qui ont fait la demande de s'installer dans le marché, ceux qui y étaient anciennement usagers, et ceux qui ont bénéficié de l'attribution des places. Les doléances adressées aux autorités locales par les victimes n'y ont rien changé. On est tenté de dire que les autorités administratives locales mêmes si elles n'ont pas participé à la prise de cette décision et sa mise en œuvre, l'ont approuvée par le silence qu'elles ont observé.

61 A ce niveau du jeu de l'autochtonie, ce sont les traditionnels étrangers à savoir les sudistes et les expatriés qui ont été victimes de la gestion en faveur des autochtones. Mais quand ils ne sont pas victimes, ils se trouvent être des observateurs d'un autre jeu à un autre niveau.

Conclusion

62 Le marché international de Parakou a été voulu par les uns et les autres comme un avantage pouvant changer le paysage environnemental urbain et promouvoir l'économie locale de la ville et même de la région. Alors que la logique principale qui est à la base de la réalisation matérielle de l'infrastructure est une logique économique, la logique de la gestion locale qui implique les principaux acteurs est sous-tendue par des idées politiques et sociales. Les problèmes du marché sont loin d'être liés à l'incapacité des commerçants de la ville à supporter les coûts des boutiques. Après maintes diminutions, les prix initiaux ont fini par être appliqués et depuis près de quatre mois il n'y a plus de troubles des usagers. Cette situation, tout de même paradoxale s'explique moins par le fait de la sensibilisation, que par le fait de ceux qui sont actuellement à la tête de la SGMP. Ils ne sont pas des gens à "destituer". Ils ont été voulus par les usagers et sont dans une certaine mesure des leurs. La conséquence est que les troubles ont changé de camp. Ce ne sont plus les usagers qui sont mécontents, ce sont les travailleurs, car les partisans / défenseurs des travailleurs sont partis, pourrait-on dire.

63 A travers ces remous et la dynamique des différents acteurs, on est arrivé à apprécier la partition jouée par chaque groupe et surtout la volonté de l'administration locale de ne pas prendre une décision qui risque de la rendre impopulaire aux yeux des usagers. Cette stratégie qui consiste à abdiquer n'est pas propre à l'administration et ses 
responsables mais est partagée par nombre de leaders politiques de la localité. C'est ce qui explique d'ailleurs le nombre élevé de groupes et de personnalités qui sont souvent appelés à la médiation quand il y a un problème. Mais ces différents médiateurs ont souvent fini par adopter une position pour les plus remuants car ils évitent eux-mêmes l'impopularité. C'est tout logiquement donc qu'au niveau local, l'option a été faîte pour d'autres logiques pourvu qu'elles arrangent les plus remuants. Face à la complexité dans laquelle s'est inscrite la gestion du marché, les stratégies mises en œuvre par les uns et les autres, les différents rebondissements et la position des pouvoirs publics et des autorités locales, il convient d'affirmer que le marché est comme la fièvre qui annonce l'infection dont souffre la ville et qui va déterminer l'état de la décentralisation future dans celle-ci. La ville est caractérisée par la facilité qu'ont les leaders à mobiliser les populations autour d'arguments ethniques. La situation apparaitt telle qu'une fois l'argument ethnique caduc, il devient presque impossible de mobiliser les acteurs autour d'une même cause. On peut se demander quelle règle déterminera le jeu politique local?

\section{BIBLIOGRAPHIE}

Assaba C. 1997. Critique de la sacralité du pouvoir. Paris : GREC.

Bailley F. 1971. Les règles du jeu Politique. Paris : PUF.

Bako-Arifari N., Laurent P.J. (eds.) 1998. Les dimensions sociales et économiques du développement local et la décentralisation en Afrique du Sud du Sahara. Bulletin APAD W 15, Hamburg, Lit Verlag.

Bako-Arifari N. 2000. "Dans les interstices de l'Etat : des courtiers en col blanc". In Bierschenk T., Chauveau U.P., Olivier de Sardan J.P. (eds.), Courtiers en développement. Paris, APAD-Karthala : 43-70.

Balandier G. 1969. Anthropologie politique. Paris : PUF.

Balandier G. 1992. Le Pouvoir sur scène. Paris : Balland.

Biersckenk T., Olivier de Sardan J.P. 1998. Les pouvoirs au villages. Le Bénin rural entre démocratisation et décentralisation. Paris : Karthala.

Gonidec P.F. 1985. L'État africain. Paris : Librairie générale de droit et de jurisprudence.

Hountondji P. 1997. Combats pour le sens, un itinéraire africain. Cotonou : Editions Flamboyant.

Hyden G, Braton M. (eds.) 1993. Gouverner l'Afrique : vers un partage des rôles. Manille : Nouveaux Horizons.

Kassibo B. (ed.) 1997. La décentralisation au Mali, état des lieux. Bulletin APAD W 14, Hamburg, Lit Verlag.

Le Bon G. 1991. Psychologie des foules. Paris : PUF.

Le Financement décentralisé : pratique et théorie. Revue Tiers-Monde, 37. 
Le Meur P.Y. 1998. "Décentralisation par le Bas et participation clientéliste au Bénin". Bulletin APAD, W 15 : 49-63. .

Lombard J. 1967. Autorités traditionnelles et pouvoirs européens en Afrique Noire. Le déclin des aristocraties sous le pourvoir colonial. Paris : Armand colin.

Muller P. 1990. Les Politiques publiques. Paris : PUF.

Neveu E. 1996. Sociologie des mouvements sociaux. Paris : La Découverte.

Olivier de Sardan J.P. 1995. Anthropologie et développement. Essai en socio-anthropologie du changement social. Paris : APAD-Karthala.

Olivier de Sardan J.P. 1998. "Chef et projet au village (Niger)". Bulletin APAD, W 15 : 65-89.

Olivier de Sardan J.P. 1993. "Le développement comme champ politique local". Bulletin APAD, W6 : 11-18.

Olson M. 1978. Logique de l'action collective. Paris : PUF.

Robineau C. 1994. "Anthropologie économique et Marché". Cahiers des sciences humaines : 23-33.

Sèhoueto L. 1997. La démocratie commence à la maison. Cotonou : Fondation Friedrich Ebert.

Spanou C. 1991. Militants et fonctionnaires : l'administration et les nouveaux mouvements sociaux. Paris : L'Harmattan.

\section{NOTES}

1.Credo béninois des temps révolutionnaires.

2.Journal Parlé du 26/09/98 sur Radio Parakou.

\section{AUTEUR}

\section{ABOU-BAKARI IMOROU}

Université Nationale du Bénin - BP 128 - Parakou Tél : (+229) 612101 -

imorouab@yahoo.com 